\title{
Effects of storage conditions of Moringa oleifera seeds on its performance in coagulation
}

\begin{abstract}
Moringa oleifera is a plant whose seeds have coagulation properties for treating water and wastewater. In this study the coagulation efficiency of Moringa oleifera kept in different storage conditions were studied. The Moringa oleifera seeds were stored at different conditions and durations; open container and closed container at room temperature $\left(28{ }^{\circ} \mathrm{C}\right)$ and refrigerator $\left(3{ }^{\circ} \mathrm{C}\right)$ for durations of 1,3 and 5 months. Comparison between turbidity removal efficiency of Moringa oleifera kept in refrigerator and room temperature revealed that there was no significant difference between them. The Moringa oleifera kept in refrigerator and room temperature for one month showed higher turbidity removal efficiency, compared to those kept for 3 and 5 months, at both containers. The coagulation efficiency of Moringa oleifera was found to be dependent on initial turbidity of water samples. Highest turbidity removals were obtained for water with very high initial turbidity. In summary coagulation efficiency of Moringa oleifera was found independent of storage temperature and container, however coagulation efficiency of Moringa oleifera decreased as storage duration increased. In addition, Moringa oleifera can be used as a potential coagulant especially for very high turbidity water.
\end{abstract}

Keyword: Water treatment; Natural coagulant; Coagulant storage; Coagulation efficiency; Moringa oleifera 